\title{
ARZombie: A Mobile Augmented Reality Game with Multimodal Interaction
}

\author{
Diogo Cordeiro, Nuno Correia \\ NOVA LINCS and Computer Science Department, \\ Faculdade de Ciências e Tecnologia, \\ Universidade Nova de Lisboa, \\ Quinta da Torre, 2829-516 Caparica, Portugal \\ Email: d.cordeiro@campus.fct.unl.pt,nmc@fct.unl.pt
}

\author{
Rui Jesus \\ M2A, ISEL - Instituto Superior de Engenharia de Lisboa, \\ Instituto Politécnico de Lisboa, \\ Rua Conselheiro Emidio Navarro \\ 1959-007 Lisboa, Portugal \\ Email: rjesus@deetc.isel.ipl.pt
}

\begin{abstract}
Augmented reality games have the power to extend virtual gaming into real world scenarios with real people, while enhancing the senses of the user. This paper describes the ARZombie game developed with the aim of studying and developing mobile augmented reality applications, specifically for tablets, using face recognition interaction techniques. The goal of the ARZombie player is to kill zombies that are detected through the display of the device. Instead of using markers as a mean of tracking the zombies, this game incorporates a facial recognition system, which will enhance the user experience by improving the interaction of players with the real world. As the player moves around the environment, the game will display virtual zombies on the screen if the detected faces are recognized as belonging to the class of the zombies. ARZombie was tested with users to evaluate the interaction proposals and its components were evaluated regarding the performance in order to ensure a better gaming experience.
\end{abstract}

Keywords-Computers and information processing, Augmented Reality, Mobile Computing, Multimodal Interaction, Games, Face Detection and Recognition.

\section{INTRODUCTION}

The world of mobile technologies is in a constant and growing popularity, becoming more ubiquitous everyday [5]. Mobile devices have increasingly demonstrated their usefulness and applicability in a daily basis, to assist users in their work, to be used in a familiar environment or to support forms of entertainment.

The world of smartphones and tablets opens up new interaction opportunities and with the evolution of the technology there are emerging devices with high computational capabilities. These devices are equipped with high-resolution cameras and other resources such as GPS and accelerometer.

As a result, the interest in implementing augmented reality applications [20] on mobile systems has increased significantly. These systems, which integrate reality with virtual elements, provide the user with an easy and safe interaction, without prior knowledge of this technology. That happens because the movements produced are natural, easy to learn and could be as simple as moving a body part or an object. Even so, there are still some limitations involving lighting issues, interaction modalities, accurate alignment between real and virtual components and the correct tracking of interesting features in the real world.
Augmented reality can be useful in any application that needs to display information not available or not directly detectable by the human senses. Therefore, by becoming visible, it is possible to increase the sense of realism and immersion of this technology. It is also common to see people using their tablets to take pictures and to play games, in various situations, on the street, on the bus and at home. Therefore, we propose a game where real elements of the scenario can be used as a form of entertainment. A game where the presence of other people in the neighborhood have a relevant role.

Accordingly to these principles, the proposed solution aims to study and develop mobile augmented reality applications, specifically for tablets. Considering that augmented reality also has the ability to improve user gaming experience, the intention is to explore interaction possibilities of augmented reality applications by developing a game for tablet devices. This game incorporates a detection and facial recognition system, which will enhance the user experience by improving the interaction of players with the real world. Integrating the augmented reality technology and real-time face recognition methods, opens up new possibilities for interaction not only for entertainment purposes but also in other areas, such as learning, simulation and planning. The game presented in this paper is not restricted to the usage of markers or head mounted displays, like the Oculus Rift [1] . Our work uses the personal device of the users, does not have environmental restrictions and can be explored in other areas of augmented reality.

This paper is organized as follows. The next section summarizes the related work. After that, it is explained the game concept, the main design issues and the relevant challenges addressed during the development of the game. The following section presents the results obtained with several tests conducted to evaluate the system and the usability of the ARZombie game. Finally, the conclusions are presented, followed by guidelines for future improvements.

\section{RELATED WORK}

This section presents related entertainment proposals using mobile and augmented platforms.

\section{A. Augmented Reality}

Augmented reality is defined [3] as a system that combines elements from the real world with virtual elements (3D) and 


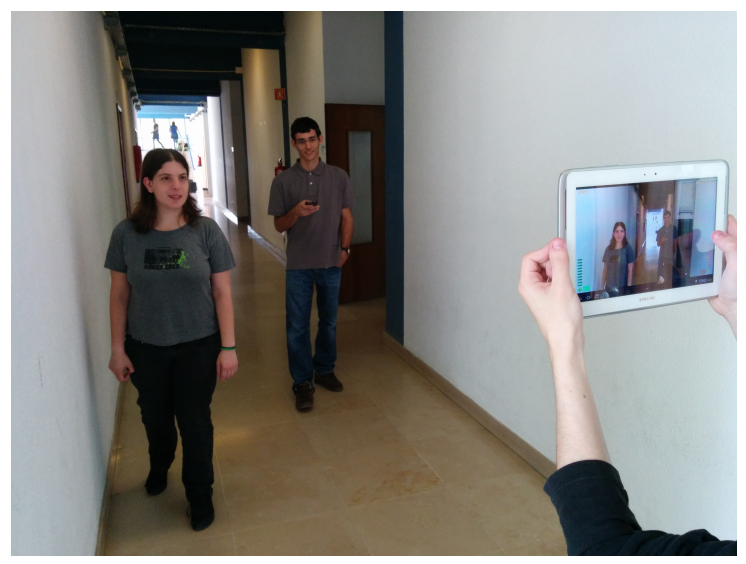

Figure 1: ARZombie: Game scenario.

that allows interactivity between objects (real and virtual) in real time. Those virtual elements allow us to display certain information, which a given user cannot detect directly only through their own senses. Thus, augmented reality changes the way we see the world, which increases the perception and interaction of a user with reality.

This type of interaction is already exploited in several areas, being used in industrial applications [21] (in the assembly, maintenance and repair of complex equipment), in medical procedures [10] (conducting tasks of high precision), in aviation [8] (presenting virtual markers that define the runway and improving image quality in low visibility situations, for example), as well as in the military [17], education [16], advertising [23] and tourism [9]. In addition to these areas, augmented reality is also used for entertainment purposes. Many of these applications are computer games.

ARQuake [26] was the first fully functional augmented reality game designed to be used in an outdoor environment. It is a first-person shooter game that allows a user to move through the real world while trying to eliminate virtual monsters. Unlike ARZombie, which uses a mobile device as a form of interaction (see Figure 1), ARQuake users are equipped with a HMD (Head Mounted Display). Despite their ability to view the world around them and observe virtual information overlaid in their helmet, players had to wear heavy devices on their head and a rigid backpack. The tracking of the user position and the head orientation is done through a hybrid system that combines GPS, compass and tracking based on optical detection of markers in the existing scenario. As a form of interaction, the user can change the current weapon or shoot the monsters that arise in the world by pressing the trigger of a tangible weapon. This form of interaction improved the gameplay of ARQuake game.

AR2Hockey [22], is another augmented reality game that allows two users to share the same environment to play air hockey. In this form of collaborative interaction, users interact with a puck, which is a virtual computer-generated object, with the objective of scoring a goal. To move the puck the player uses a mallet, which can be observed using optical see-through HMDs. As a form of hybrid tracking, the HMD uses magnetic sensors to capture the orientation of the head and a camera to detect physical markers placed in the game area in order to compensate for errors arising from the magnetic sensor readings. One conclusion drawn from this system is related to the user experience, that is much better if registration is accurate and fast enough. Therefore, misalignment between the physical and virtual space, caused by the delay in the calculation and representation of virtual elements, should be minimized.

\section{B. Handheld Devices}

With the evolution of technology, handheld devices have emerged with high computational capacity, equipped with high-quality color displays, high resolution digital cameras, and real-time hardware-accelerated 3D graphics [25]. They also integrate additional sophisticated sensors like accelerometer and GPS. Therefore, it is possible to see that, with such characteristics, these devices could be one of the main platforms in the development and commercialization of augmented reality applications.

"ARhrrrr" [18] is an augmented reality shooter for mobile camera-phones. The game allows the user to move around a table and the objective of the player is to kill zombies that are getting closer to the civilians. ARhrrrr uses video-based tracking to detect "skittles", which may be used as bombs, and to detect the game table image, which will display the virtual city. This game has similarities with ARZombie but, in this case, the player is limited to the movement around a table. Unlike the ARZombie game (that aims to achieve a good gameplay by recognizing faces), the player needs to constantly detect specific 2-D pictures or markers to be able to play and view all virtual elements.

Another game that revolves around the zombies concept is the Moxie Mayhem Augmented Reality Game [2]. It uses markers placed on the head of the players as a form of interaction. Through these markers, detected with the cameras of the handheld devices, it is possible to find out if a player is a zombie or a human being. To play this game all the players (regardless of whether they are zombies or not) need to put QRCodes on their heads, as opposed to the ARZombie game, which is able to just capture and store images from the players that belong to the zombies' group.

Due to the ubiquitous use of mobile devices (smartphones and tablets) and the characteristics of this type of equipment, it becomes easier to implement augmented reality games in real environments [27]. However, it is necessary to solve some challenges, especially in relation to the interaction with computer generated virtual objects.

\section{Video-based Tracking Techniques}

With the availability of fast processors that capture video frames efficiently, this type of technique becomes extremely popular [24]. Being one of the most used [32] (when compared with sensor-based and hybrid techniques) this technique can determine the position of the camera relative to real-world objects through image processing methods. Video-based techniques are able to detect features in the environment. These features can be artificially created by using markers (in which a pattern is drawn) or can be natural patterns like human faces, the latter being constantly used in ARZombie. 
One possible approach consists of finding correspondence between the characteristics of the captured image and its actual coordinates. This operation can be done by using markers that allow the graphics system to know the spatial coordinates and orientation of the objects from the point of view of the user.

Some systems are built to analyze the contours and edges in order to detect predefined models. The contours are the most used features because they are robust to light changes. One example of the usage of this method is to identify and track, in real-time, known planar patterns, which consist of a set of corners [19]. The main idea involves the continuous monitoring of predefined patterns where, from the analysis of each video frame, are detected not only the four outer corners of the patterns as well as the interiors, in real time.

Other example [30] describes a new approach for detecting and tracking multiple moving objects in video images acquired by a static camera. Unlike many augmented reality applications, this system, as well as the ARZombie game, does not use extra tracking devices, only algorithms based on image and video processing. The tracking system is able to track multiple objects simultaneously. These PHOs (place holder objects) are placed on a table and can be moved arbitrarily, allowing them to be rotated independently of the viewing range of the camera, which is oriented to the table.

\section{Face Detection and Recognition}

It is interesting to analyze how facial detection and recognition can be implemented in augmented reality applications. To do this, it is necessary to understand how face detection and recognition methods are related and how their limitations can influence the systems in which they are integrated. As described by W. Zhao et al. [31], facial recognition systems involve three key steps: (1) face detection, (2) feature extraction and accurate normalization of faces and finally the (3) identification and/or verification of faces.

1) Face Detection: Face detection consists in finding faces on a particular image. Face detection plays a very important role in the area of human-computer interaction, being the first step in various processes of recognition (faces, facial features or expressions) [7]. To build fully automated systems it is important that the detection methods implemented are efficient and robust, as the human face can be seen as a dynamic object, which has a high degree of variability [12]. There are other factors, in addition to the above, which also make more difficult the face detection task. An example is the presence of hair on the face, makeup, beard or mustache or even wearing glasses or hats.

There are several approaches to implement detection based on characteristics. Among others, there are methods, which detect faces using the information collected from grayscale images. The Viola-Jones detection framework [29] is used to detect the face and eyes in the frontal face images, based on a cascade of classifiers. Some are based on the knowledge of the contours in the images. In one of the approaches [33], images are processed to extract its contours accurately. After the analysis of the image gradient, the system will verify the presence of the eyes, nose and mouth as a form of detection. Other methods use color as a way to detect the skin of the various faces. One method [11] adds the detection of the skin and the calculation of the face orientation in order to improve the detection rate. Experimental results showed that these two features improve the overall performance of the system used, and it was capable of detecting more faces correctly (true positives) and had a lower rate of false positives.

2) Face Normalization: Normalization is an intermediate step applied after the process of detecting faces and before starting facial recognition. Since various regions of the detected faces may have different characteristics (essentially different scale, orientation and lighting), all detected faces are normalized. A. Lameira et al. [15] uses the normalization phase to extract the area of an object and set it to a predefined format. To achieve this goal, without distorting the object area, the following steps are performed: Firstly, a square region is created. Then the area of the object is placed within that region, filling the remaining space with black pixels. Finally, the object image is converted to a default resolution, for example, 90 by 90 pixels.

3) Face Recognition: It is clear that despite the current face recognition systems had achieved a certain level of maturity, their success is still limited by the conditions imposed by many real applications [31]. For example, there are problems in faces detected in outdoor environment images where the illumination conditions are not always the same. The orientation of the people (face) also remains a problem, still with no ideal solution. In other words, current systems are still far from being compared with the recognition capability of the human perception system.

A major goal of face recognition algorithms is to deal with certain variations that may affect faces in the images [6]. Ideally, a method of face recognition should be able to recognize a face despite these variations, but in practice this does not happen. If there is much noise in the image, the performance of face recognition methods decreases significantly. An image or a detected face contains noise if there are variations that are classified into three distinct categories: (1) if there are significant variations in illumination, (2) if the selection of the face region to be identified is inaccurate or (3) if the face is not in a frontal position relative to the camera. These are the main issues that have to be considered in the ARZombie game.

\section{ARZOMBIE}

This section presents the research questions, the game concept and the interaction possibilities that it opens. Issues such as tracking, registration, mobility and lighting will be discussed.

\section{A. Research questions}

Despite the considerable advances made in the area of augmented reality, there are interaction limitations that still remain with the current technology, which in the ARZombie game context represent the main research challenges that must be discussed and addressed. The main challenges are related to tracking, registration, mobility and illumination problems. 


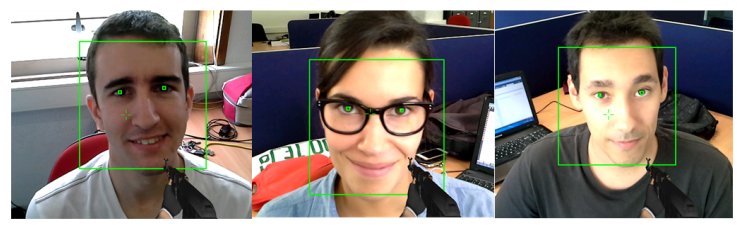

Figure 2: ARZombie tracking some faces.

1) Tracking: The main difficulties related to tracking people in real time are due to the complexity in detecting key patterns (faces in ARZombie) in heterogeneous environments, which are constantly changing their characteristics because the user is moving. These difficulties include the detection of moving objects that can become partially hidden or their appearances can change due to the variation of brightness or if there is noise in the captured image.

In vision-based tracking systems, the use of markers in the environment helps the detection and processing of captured images, which increase the robustness and reduce computational requirements. Nevertheless, the markers need maintenance and the range to which markers are recognized by the system is limited, providing relevant information only when they are detected. Thus, tracking methods based on markers are not scalable, especially in outdoor scenarios.

ARZombie takes advantage of existing natural resources in the environment. Based on a predefined model of the human face (Figure 2), the range of the tracking area using these features is extended and it does not need prior knowledge of the location of the player.

2) Registration: Many studies have also focused on the issue of proper alignment with elements of virtual reality, such as registration [4]. While significant progress has been made it is still far from being a solved problem.

The augmented reality systems attempt to ensure full consistency between real and virtual objects. A small error in the overlap of objects may cause a noticeable misalignment between these two realities, compromising the illusion that the two worlds coexist.

The determination of the exact position and orientation of the user is especially important in these applications, so as to appear more realistic. Without a proper alignment, many augmented reality applications will not be accepted by the users [3]. Consider, for example, a surgeon performing a biopsy. In such situations, an exact alignment between the tumor and the virtual object is required.

In ARZombie, computer-generated creatures are placed over the head of the recognized faces, based on their size and orientation, determined by the face detection component (Figure 3). For this reason, face detection is a key operation in the ARZombie game.

3) Mobility: Another issue in ARZombie game is related to its playability. The user needs to be in motion. In almost all virtual reality systems, the user is not encouraged to move, staying in one place during real-world usage of these applications [3]. However, in certain applications of augmented

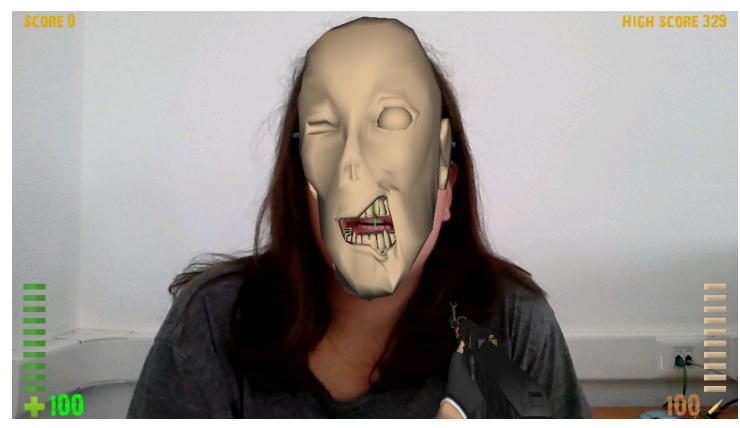

Figure 3: Result of the alignment between the face of the person and the virtual model.

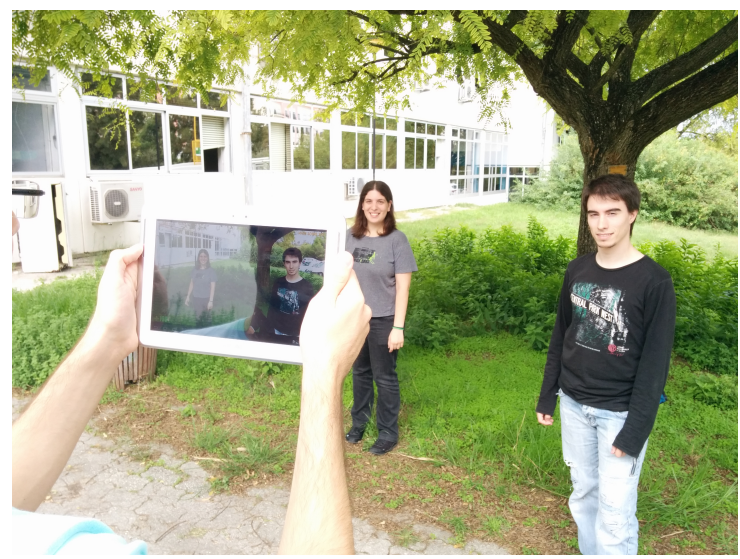

Figure 4: ARZombie game being played outdoor.

reality it is necessary to provide support for a user that walks freely through the real world.

Mobile devices such as HMD required the use of a helmet and a backpack to carry the computer, camera, tracking sensors, the display screen and batteries. These devices became uncomfortable for users due to the weight of the components.

The game presented in this paper is intended to be played in a tablet, which is a more personal device and very light and slim, when compared with the previously mentioned devices. With the ability to detect human faces, this game has no location restrictions. This means that it is not necessary to have prior knowledge of the location of the player, the scenarios where the user goes to, nor the various zombies that appear in front of the player. In Figure 4, we can observe the use of this game in a outdoor location and, in Figure 5, the player uses his tablet in order to play inside a building.

4) Lighting: The ambient lighting is an important component when using augmented reality applications, especially in the generation of virtual objects [14]. Used for applications in familiar environments it is possible to correctly model the light and project it onto virtual objects. In outdoor applications, the display of the device must work properly in a wide variety of lighting conditions [4]. Thus, ARZombie takes that into consideration and demonstrates to the user that the quality of the faces collected from each person plays an important part, for a more efficient use of the game. In unexpected situations, we still have some errors, were some faces are not detected 


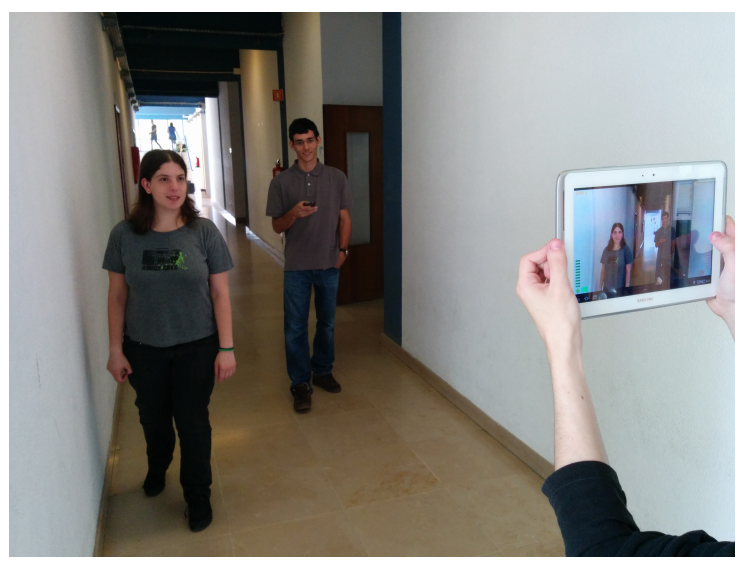

Figure 5: ARZombie game being played indoor.

(as described in the evaluation section), which are relevant to the playability of the game.

\section{B. Game Concept}

After developing the essential components of this research, the ARZombie game was implemented as a way to integrate and evaluate them. ARZombie users are able to interact with the integration between augmented reality and facial recognition. The goal of this game is to ensure that the users eliminate a great number of potential zombies, allowing them to receive more points. At each level, creatures become more dangerous and inflict more and more damage to the player. If the player is attacked continuously, they will eventually lose the game. To avoid being attacked, the player has to eliminate the zombies, by shooting their heads, with the help of the tablet. In order to detect the zombies, the goal is to capture human faces so that the tablet analyzes whether they are zombies or not.

By combining augmented reality with face recognition, we give the user a fun and adventurous form of entertainment. Since the players can define who are the zombies, the interaction with their friends and family can lead to an engaging and fun gameplay.

The game story, that explains the context of the game, is presented to the player as follows: "Recently, an artificial virus was created in a university laboratory. Although it was developed by accident, several people are already infected and have been slowly turning into zombies. Despite the changes of the virus in the human body they are not yet visible to the naked eye. There is mobile equipment prepared to analyze and detect whether each person is already infected or not. Since there is still no cure, it is necessary to eliminate all the zombies in order to control this terrible plague. To finally destroy the zombies the only solution is to damage their brain, which can be achieved by aiming the gun to the head of the zombies."

\section{Gameplay and User Interface}

The game idea is to connect the players, the people and the real world through virtual elements. The ARZombie game has the following interaction (game loop):

- $\quad$ The player walks through a physical space;

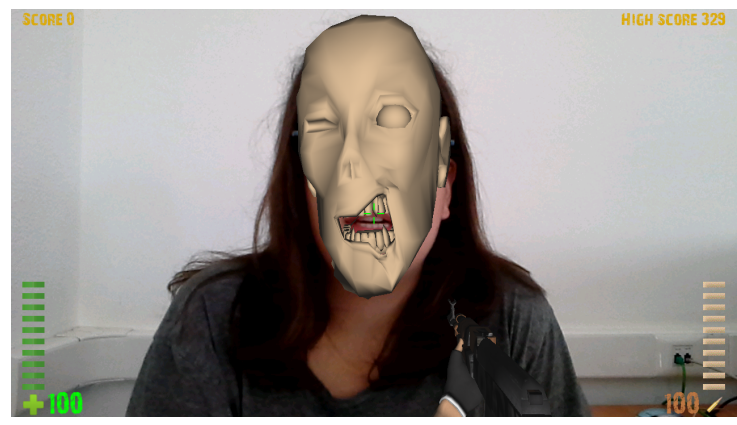

Figure 6: Detection of a zombie.

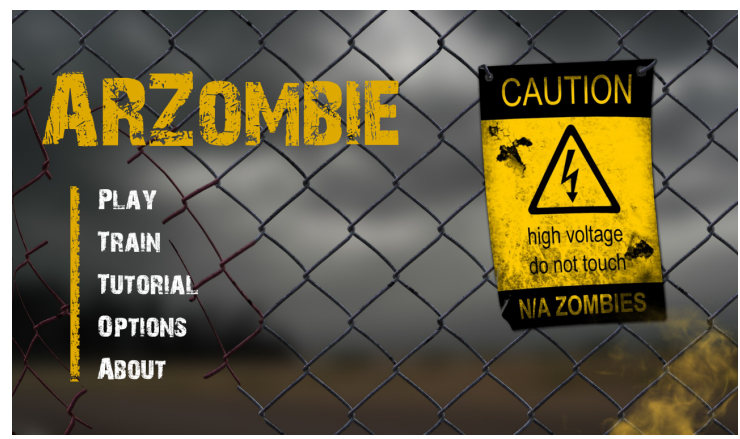

Figure 7: ARZombie main menu.

- Through the video camera, integrated in the handheld device of the player, the user will analyze the people who are in his/her vicinity;

- The game engine verifies if the detected people are zombies or not;

- If someone is recognized by the system, a computer generated virtual creature is displayed on the screen superimposed to his/her face, as we can see in Figure 6.

After the application starts (Figure 7), the game display offers the user the possibility to define (train) his friends as zombies. The tutorial section explains all the game mechanics and forms of interaction. The options menu allows the user to set on or off the sound and vibration and set the fire mode of the game. The fire mode can be of two types: (1) manual, the user have to touch the screen in order to fire the gun, and (2) automatic, the game engine fires the gun automatically if the zombie appears on the screen.

If the user has already defined some zombies, he/she is able to start playing. The goal of the player is to eliminate the zombies by shooting them in the head, in order to earn points. The game display (Figure 8) shows the points acquired during the current game as well as the high score of the user on the top of the screen. While shooting, the player can visualize the gun firing and, when the zombie attacks the user, blood animation is displayed on the screen. On the bottom it is presented the remaining life of the player, the weapon, the remaining bullets and the current bonus (if activated). The bonus is a box, that appears randomly and momentarily on the screen, that helps the user by allowing him to recover health, by protecting the 


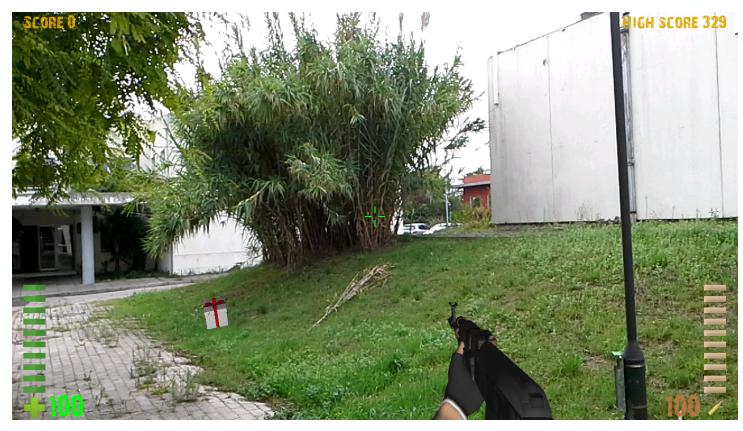

Figure 8: ARZombie game interface.

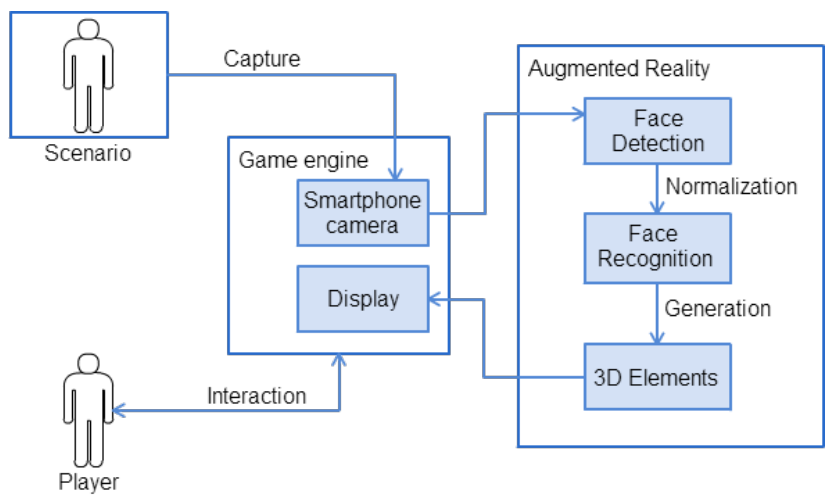

Figure 9: Diagram of the main components of the game.

player from zombie attacks or improving the quality of the gun.

\section{Architecture}

The game includes several components, as shown in Figure 9 and described in the following sections.

1) Face detection: Once the game starts, the camera begins to capture the scene. The images obtained are analyzed by the facial detection component that will check for faces. This is made with the Viola-Jones object detection algorithm that is capable of high speed image processing while achieving good detection rates [29]. With this method we are able to detect both the face and the eyes of the humans and the zombies. In order to test the performance of the game, the face detection component works in one of three ways: (1) face detection without eye detection, (2) face detection and eye detection using haar cascades (mentioned previously with the ViolaJones object detection algorithm) and (3) face detection and eye detection using edges. The eye detection process using edges (Figure 10), applies a blur effect on the detected face, as a way of reducing the noise present in the image. The various edges of the face region are detected and dilated to generate more generalized contours. The contours are them collected from each eye region to determine the approximate center of each eye.

2) Face normalization: If someone is detected, the recognition system must verify if that person belongs to the group of zombies or not. But before, the faces found are normalized, in order to increase the accuracy of the next component.

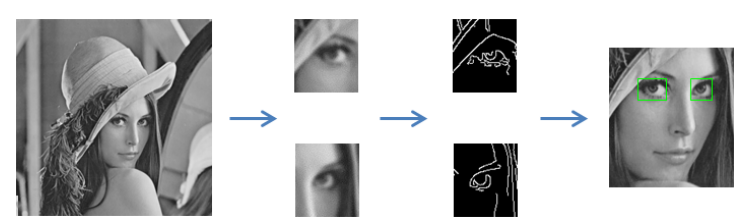

Figure 10: Eye detection process by edges.

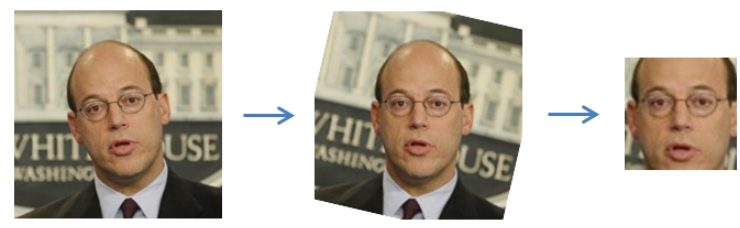

Figure 11: Face normalization process.

The normalization process will reduce the face region to a predefined size (70x70 pixels) and, if it is using the information about the eyes, to a predefined orientation, as we can see in Figure 11.

3) Face recognition: Before the game even starts the user has the opportunity to define who the zombies are. In ARZombie, the system has to learn an Eigenfaces [28] model on a given set of images. Those images (various faces of all the zombies) are captured and labeled as zombies. With those images and with the help of the OpenCV primitives, we can finally train them on the given dataset (the face images and labels).

During the game, this component is executed in real time. The detected faces are analyzed and compared with the existing trained model stored in the application, in order to determine whether, to the game engine, a particular person is a zombie or not. The Figure 12 explains the face recognition process.

4) $3 D$ elements: If the game recognizes zombies, the function of the augmented reality component is to generate virtual elements correctly aligned with the face in question and present them on the tablet screen. These 3D elements were previously parsed and when they need to appear on the screen, they are scaled, rotated and translated in order to ensure realism as much as possible.

\section{EVALUATION}

This section describes interaction tests with users and system tests to evaluate the performance of the system.

\section{A. System tests}

The evaluation of the system is divided into two sections: (1) face detection and (2) face recognition. To test the algorithms used in both components the public database "Labeled Faces In The Wild" [13] was used. 


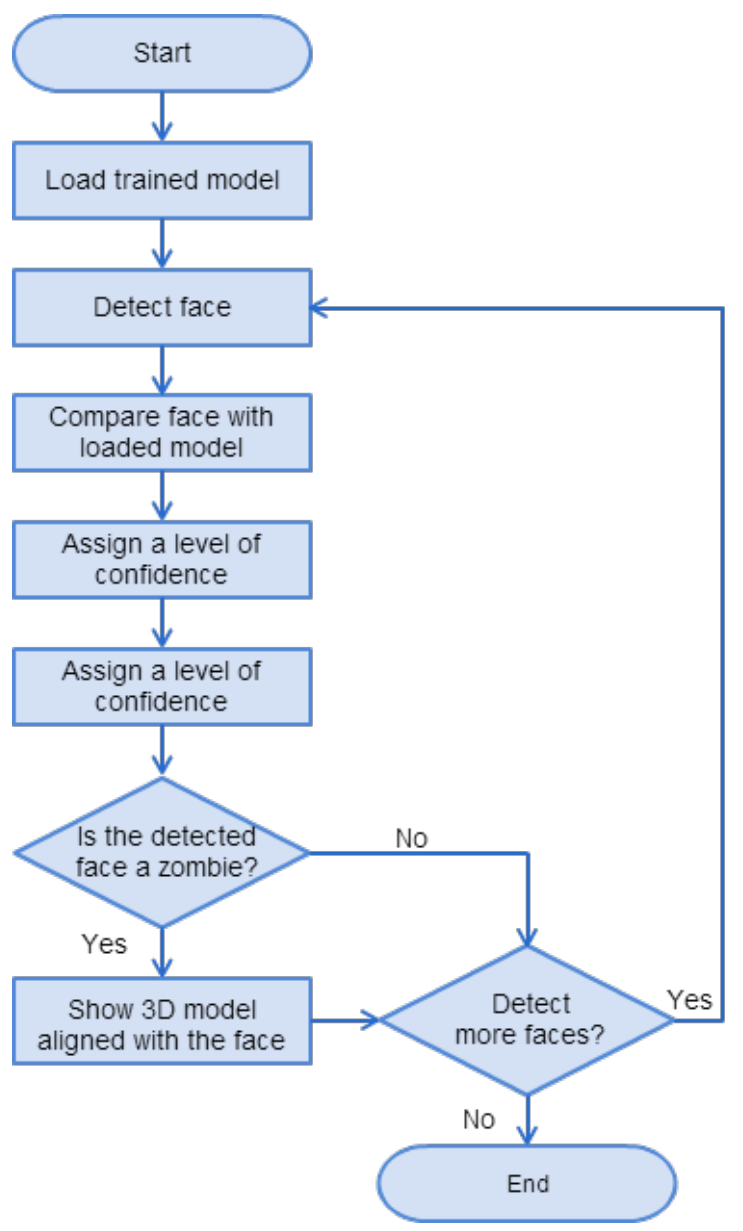

Figure 12: Face recognition process.

1) Face detection: With the ARZombie game running, a set of 105 images were used to evaluate this component, that involves the face detection system plus the three algorithms implemented (eye detection using haar cascade, using edges and without eye detection). It was also collected information regarding the approximate minimum distance (in pixels) at which each face and both eyes were detected. Tables I shows the results obtained by the face detection methods.

TABLE I: FACE DETECTION RESULTS

\begin{tabular}{|c|c|c|c|c|c|c|}
\hline & \multicolumn{2}{|c|}{ Haar cascade } & \multicolumn{2}{|c|}{ Edges } & \multicolumn{2}{|c|}{ No eyes } \\
\hline & Faces & $\%$ & Faces & $\%$ & Faces & $\%$ \\
\hline Detected & 104 & $99 \%$ & 86 & $82 \%$ & 105 & $100 \%$ \\
\hline Not detected & 1 & $1 \%$ & 19 & $18 \%$ & 0 & $0 \%$ \\
\hline
\end{tabular}

As we can see, it was registered, for each tested algorithm, the number of faces that they detected successfully, or not. We can verify that increasing the eye detection complexity, the ability of the algorithms to detect all faces (and both eyes) decreases. Another conclusion drawn from these tests is that the algorithm that does not detect eyes is able to work properly at a greater distance. The one using the haar cascade also shows a good performance, but in terms of distance, the player needs to be closer to the other people, so that both eyes can be detected. We can also see that the algorithms that use eye detection behave less efficiently and reduce the game performance (due to the amount of processing required from the device). Nevertheless, the existence of these algorithms cannot be ruled out, because they might improve the hit rate of the facial recognition component (one of the goals of this game).

2) Face recognition: Since the component of facial recognition determines whether a person belongs to the group of zombies or not, then its performance, during the game, should be the best possible. Therefore, this component was subjected to a set of tests to try to find out the quickest and accurate way to identify the zombies. For each of the three algorithms implemented, the 2-fold cross-validation technique was applied, in order to measure the predictive performance of a model, from a data set. The data set is separated into two sets, the training set and the testing set. About 180 images (30 images from 6 distinct people) were collected to carry out the tests. Of each person, $50 \%$ of the images were used in the training process and the other half was used to evaluate the face recognition algorithm. Trained faces were labeled as zombies. Since 2 folds was used, the testing set was then used in the training process and the training set was used in the recognition process. For the first test only the facial detection was used, before the normalization process. This means that the objective of this test was to understand how the absence of the eye detection component would influence the performance of face recognition, since the system does not rotate the detected faces to a predefined orientation. For the second test the eye detection algorithm that uses edges was added. It this method it was introduced, in the normalization process, the rotation of the faces. Then, for the final test, it was used a more precise method of detecting both eyes, using haar cascade, replacing the previous one. Tables II, III and IV show the results obtained, respectively.

TABLE II: FACE RECOGNITION RESULTS WITHOUT EYE DETECTION.

\begin{tabular}{cc|c|c}
\hline \multirow{2}{*}{ No eye detection } & \multicolumn{2}{c}{ Classification } \\
\cline { 2 - 4 } Group & Not zombie & Zombie \\
\cline { 2 - 4 } & Not Zombie & 22 & 8 \\
\cline { 2 - 4 } & Zombie & 65 & 85 \\
\hline
\end{tabular}

TABLE III: FACE RECOGNITION RESULTS WITH EDGES.

\begin{tabular}{c|c|c|c}
\hline \multirow{2}{*}{ Edges } & \multicolumn{2}{c}{ Classification } \\
\cline { 2 - 4 } Group & Not zombie & 22 & 8 \\
\cline { 2 - 4 } & Zombie & 62 & 88 \\
\hline
\end{tabular}

TABLE IV: FACE RECOGNITION RESULTS WITH HAAR CASCADE.

\begin{tabular}{c|c|c|c}
\hline \multirow{2}{*}{ Haar cascade } & \multicolumn{2}{c}{ Classification } \\
\cline { 2 - 4 } Group & Not zombie & Zombie \\
\cline { 2 - 4 } & Not zombie & 20 & 10 \\
\cline { 2 - 4 } & Zombie & 18 & 132 \\
\hline
\end{tabular}

We can observe that the various algorithms were able to achieve satisfactory results (taking into account the high 
degree of variability of several tested images). In terms of performance, the algorithm that uses haar cascade obtained the best results with an accuracy of approximately $84 \%$ and the one based on edges had an accuracy of approximately $61 \%$. The worst performance was obtained by the algorithm that does not detect the eyes, with an accuracy of approximately $59 \%$. By comparing the tables, it is possible to see that reducing the precision and complexity of the eye detection algorithms, decreases the accuracy of the face recognition component. So, we can conclude that determining the correct position of both eyes, and applying the rotation in the normalization process, is essential for the proper functioning of facial recognition algorithms. With these tests, we demonstrated that the implemented face recognition component works very well, having the ability to be used in the ARZombie game or any other system. The results show that the integration of this system in the augmented reality system is reliable and can be used as an alternative to the existing methods (markers, for example).

\section{B. User tests}

This section describes the evaluation process of the game developed, made by several users. Aiming to ensure overall user satisfaction, the main focus of attention was given to the following aspects:

- Accessibility and usability (interface and navigation within the game);

- $\quad$ Easy identification of virtual elements;

- Correct adjustment of the various factors of the game;

- Analysis of the reactions of the players;

The tests were performed on a population of 15 voluntary participants, ranging in age from 22 to 26 years old. The test sessions were conducted individually for each user. Before starting to use the application, users were informed about the application and the objectives of the test. The users were encouraged to "think aloud" and tell what was going through their minds while using the application. Afterwards, participants were asked to fill in a questionnaire, indicating their level of agreement with each statement, by circling a value on a 5-point Likert-type scale, with a response of 1 (one) meaning "totally disagree" and a response of 5 (five) meaning "totally agree". The questionnaire comprised questions regarding how easy it was to learn the mechanics of the game, their movement through the scenario, how useful was the information presented on the screen and how well was the user able to kill the zombies and activate the bonus, as well as suggestions and comments.

The experimental feedback was evaluated through several sections of the questionnaire comprising some statements each. Table $\mathrm{V}$ shows the statements included in the questionnaire.

From the usability tests we can conclude that the users were satisfied with the ARZombie game (Figure 13), not only because of the type of interaction introduced, but also because it was not difficult for them to interact with the game and the application was well structured, in their opinion. Regarding the physical interaction of the player with the game, it can be concluded that the users moved easily through the scenario
TABLE V: MAIN STATEMENTS INCLUDED IN THE QUESTIONNAIRE.

\begin{tabular}{ll}
\hline & Learning: \\
\hline 1 & It is easy to learn to play. \\
2 & It is easy to interact with the game. \\
3 & Easy adaptation to the game. \\
4 & The application is well structured. \\
& Movement: \\
\hline 5 & The user is able to move freely through the scenario. \\
6 & Interactions with the game are natural. \\
\hline \multicolumn{2}{c}{ Information: } \\
\hline 7 & Visual aspects involve the user during the game. \\
9 & Sound aspects involve the user during the game. \\
9 & The user is aware of the information shown on screen. \\
\hline 10 & Elements visible on the screen are distracting. \\
\hline 11 & Zombies are easily detected. \\
12 & Bonus is easily detected when appear in the display. \\
\hline & Control: \\
\hline 13 & User is able to eliminate zombies without problems. \\
14 & User is able to activate the bonus without problems. \\
\hline &
\end{tabular}

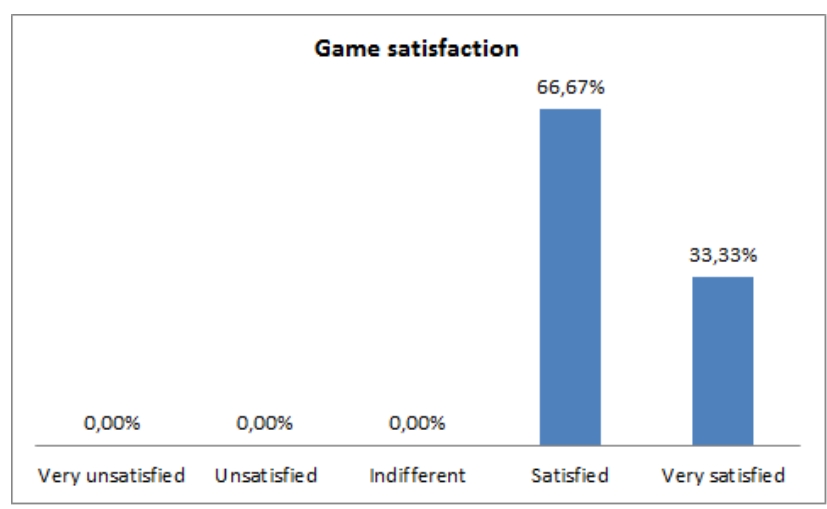

Figure 13: User satisfation.

(Figure 14) and that the interactions with the game were natural, in terms of walking towards the zombies, shooting the gun and activating the bonuses. Both the sound aspects and all the information shown on the display were not distracting (Figure 15) and helped in the interaction of the users with the game, informing them of the presence of zombies, bonuses, their remaining life and ammunition. Results also indicate that users had no problem identifying and activating bonuses and detecting which person was a zombie (Figure 16). It was very easy for the users to aim the gun to the head of the zombie, thereby eliminating them without any problems.

\section{CONClusion AND Future Work}

This paper describes ARZombie, an augmented reality game. Our proposal is to use facial detection and recognition as an interaction modality, in this case a way of tracking zombies. With this platform and interaction techniques we are able to develop functional games with multimodal interaction. Tests were performed to evaluate the usability and performance of the game. From the usability tests results we can conclude that the majority of users were satisfied by this type of game interaction. Through this game we ensure that the interaction and integration of these technologies (augmented reality and face recognition) is a feasible option, which can be used not only for entertainment purposes, but also as a mechanism to be explored in various areas. From the system tests results, 


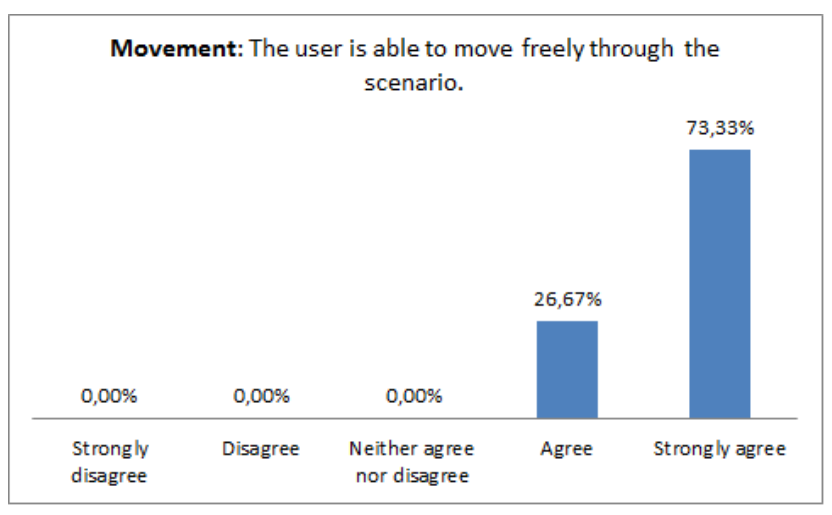

Figure 14: User mobility.

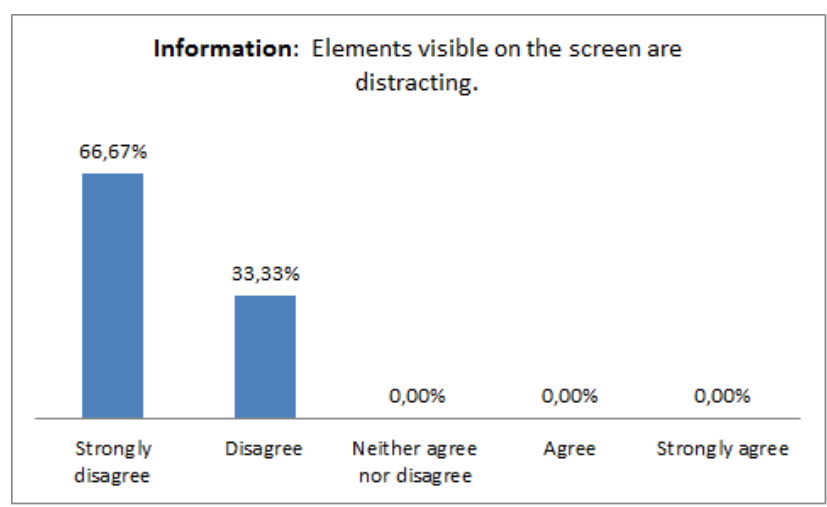

Figure 15: Distracting information.

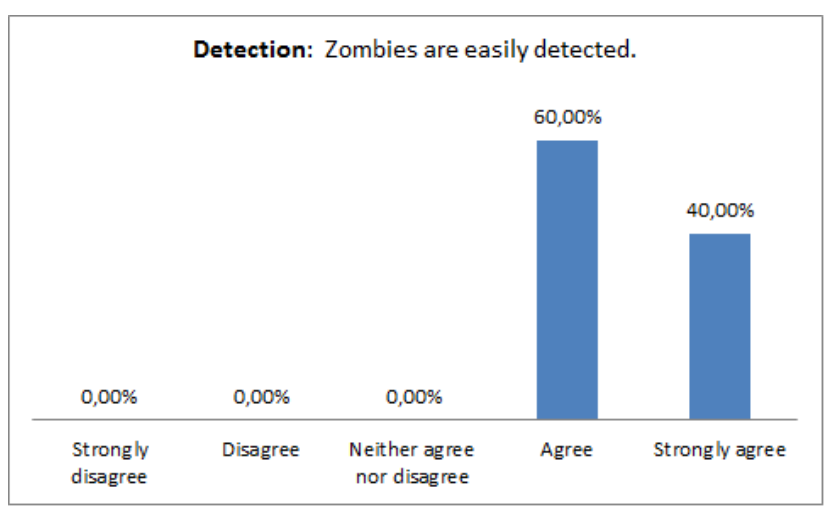

Figure 16: Zombie detection.

we can conclude that by adding eye detection prior to the normalization process increases the accuracy of the recognition system, but, in order to detect both eyes, the player has to be closer to other people. Regarding future work, it would be useful to develop a dynamic algorithm that had the ability to incorporate the three algorithms developed for the ARZombie game, adapting its functioning according to the proximity between the player and other people. This approach could potentially improve the efficiency and accuracy of both face detection and recognition. It is also important to explore other ways to score in this game. Instead of eliminating the zombies by shooting a gun, we could develop a more friendly way of dealing with potential enemies. Finally, it would be interesting to explore the approach presented in this article with Google Glass technology.

\section{REFERENCES}

[1] Next-generation virtual reality technology. http://www.oculus.com/dk2/, Nov. Last access: 10/Nov/2014.

[2] J. Archer. Zombie augmented reality game. http://vimeo.com/48006574, Last access: 19/Jul/2012.

[3] R. T. Azuma. A survey of augmented reality. Presence: Teleoperators and Virtual Environments, 6(4):355-385, Aug. 1997.

[4] R. T. Azuma. The challenge of making augmented reality work outdoors. In In Mixed Reality: Merging Real and Virtual, pages 379390. Springer-Verlag, 1999.

[5] R. Ballagas, J. Borchers, M. Rohs, and J. G. Sheridan. The smart phone: A ubiquitous input device. IEEE Pervasive Computing, 5(1):70-77, Jan. 2006.

[6] S.-A. Berrani and C. Garcia. On the impact of outliers on highdimensional data analysis methods for face recognition. In Proceedings of the 2Nd International Workshop on Computer Vision Meets Databases, CVDB '05, pages 43-49, New York, NY, USA, 2005. ACM.

[7] M. V. Choudhari, M. S. Devi, and P. Bajaj. Face and facial feature detection. In Proceedings of the International Conference \&\#38; Workshop on Emerging Trends in Technology, ICWET '11, pages 686689, New York, NY, USA, 2011. ACM.

[8] F. D. Crescenzio, M. Fantini, F. Persiani, L. di Stefano, P. Azzari, and S. Salti. Augmented reality for aircraft maintenance training and operations support. IEEE Computer Graphics and Applications, 31(1):96-101, 2011.

[9] F. Fritz, A. Susperregui, and M. T. Linaza. Enhancing cultural tourism experiences with augmented reality technologies. 6th International Symposium on Virtual Reality, Archaeology and Cultural Heritage (VAST), November 2005.

[10] H. Fuchs, M. A. Livingston, R. Raskar, A. State, J. R. Crawford, P. Rademacher, S. H. Drake, and A. A. Meyer. Augmented reality visualization for laparoscopic surgery. In In Proceedings of the First International Conference on Medical Image Computing and ComputerAssisted Intervention, pages 934-943. Springer-Verlag, 1998.

[11] F. Grangeiro, R. Jesus, and N. Correia. Face recognition and gender classification in personal memories. In Proceedings of the 2009 IEEE International Conference on Acoustics, Speech and Signal Processing, ICASSP '09, pages 1945-1948, Washington, DC, USA, 2009. IEEE Computer Society.

[12] E. Hjelms and B. K. Low. Face detection: A survey. Computer Vision and Image Understanding, 83(3):236 - 274, 2001.

[13] G. B. Huang, M. Ramesh, T. Berg, and E. Learned-Miller. Labeled faces in the wild: A database for studying face recognition in unconstrained environments. Technical Report 07-49, University of Massachusetts, Amherst, October 2007.

[14] D. Koller, G. Klinker, E. Rose, D. Breen, R. Whitaker, and M. Tuceryan. Real-time vision-based camera tracking for augmented reality applications. In Proceedings of the ACM Symposium on Virtual Reality Software and Technology, VRST '97, pages 87-94, New York, NY, USA, 1997. ACM

[15] A. Lameira, R. Jesus, and N. Correia. Real-time object recognition using mobile devices. In Proceedings of the 13th International Conference on Human Computer Interaction with Mobile Devices and Services, MobileHCI '11, pages 687-690, New York, NY, USA, 2011. ACM.

[16] K. Lee. Augmented Reality in Education and Training. TechTrends, 56(2):13-21, Jan. 2012.

[17] M. A. Livingston, L. J. Rosenblum, D. G. Brown, G. S. Schmidt, S. J. Julier, Y. Baillot, J. E. S. II, Z. Ai, and P. Maassel. Military applications of augmented reality. In B. Furht, editor, Handbook of Augmented Reality, pages 671-706. Springer, 2011.

[18] B. MacIntyre. Arhrrrr! http://blairmacintyre.me/project/arhrrrr/.

[19] S. Malik, C. McDonald, and G. Roth. Hand tracking for interactive pattern-based augmented reality. In Proceedings of the 1st International Symposium on Mixed and Augmented Reality, ISMAR '02, pages 117-, Washington, DC, USA, 2002. IEEE Computer Society. 
[20] A. Mulloni, A. Dünser, and D. Schmalstieg. Zooming interfaces for augmented reality browsers. In Proceedings of the 12th International Conference on Human Computer Interaction with Mobile Devices and Services, MobileHCI '10, pages 161-170, New York, NY, USA, 2010. ACM.

[21] L. Naimark and E. Foxlin. Circular data matrix fiducial system and robust image processing for a wearable vision-inertial self-tracker. In Proceedings of the 1st International Symposium on Mixed and Augmented Reality, ISMAR '02, pages 27-, Washington, DC, USA, 2002. IEEE Computer Society.

[22] T. Ohshima, K. Satoh, H. Yamamoto, and H. Tamura. Ar2 hockey: A case study of collaborative augmented reality. In Proceedings of the Virtual Reality Annual International Symposium, VRAIS '98, pages 268-, Washington, DC, USA, 1998. IEEE Computer Society.

[23] Snickersenergy. Snickers zombie transformation video. https://www. youtube.com/watch?v=3jdFFVPS1q0, Last access: 23/Dez/2013.

[24] R. Subbarao, P. Meer, and Y. Genc. A balanced approach to 3d tracking from image streams. In Proceedings of the 4th IEEE/ACM International Symposium on Mixed and Augmented Reality, ISMAR '05, pages 7078, Washington, DC, USA, 2005. IEEE Computer Society.

[25] G. Takacs, V. Chandrasekhar, N. Gelfand, Y. Xiong, W.-C. Chen, T. Bismpigiannis, R. Grzeszczuk, K. Pulli, and B. Girod. Outdoors augmented reality on mobile phone using loxel-based visual feature organization. In Proceedings of the 1st ACM International Conference on Multimedia Information Retrieval, MIR '08, pages 427-434, New York, NY, USA, 2008. ACM.

[26] B. Thomas, B. Close, J. Donoghue, J. Squires, P. D. Bondi, and W. Piekarski. First person indoor/outdoor augmented reality application Arquake. Personal Ubiquitous Comput., 6(1):75-86, Jan. 2002.

[27] B. H. Thomas. A survey of visual, mixed, and augmented reality gaming. Comput. Entertain., 10(3):3:1-3:33, Dec. 2012.

[28] M. Turk and A. Pentland. Eigenfaces for recognition. J. Cognitive Neuroscience, 3(1):71-86, Jan. 1991.

[29] P. Viola and M. J. Jones. Robust real-time face detection. Int. J. Comput Vision, 57(2):137-154, May 2004

[30] C. Yuan. Simultaneous tracking of multiple objects for augmented reality applications. In Proceedings of the Seventh Eurographics Conference on Multimedia, EGMM'04, pages 41-47, Aire-la-Ville, Switzerland, Switzerland, 2004. Eurographics Association.

[31] W. Zhao, R. Chellappa, P. J. Phillips, and A. Rosenfeld. Face recognition: A literature survey. ACM Comput. Surv., 35(4):399-458, Dec. 2003.

[32] F. Zhou, H. B.-L. Duh, and M. Billinghurst. Trends in augmented reality tracking, interaction and display: A review of ten years of ismar. In Proceedings of the 7th IEEE/ACM International Symposium on Mixed and Augmented Reality, ISMAR '08, pages 193-202, Washington, DC, USA, 2008. IEEE Computer Society.

[33] Q. Zhu and J. Chen. A new approach for rotated face detection. In Proceedings of the Ninth ACM International Conference on Multimedia MULTIMEDIA '01, pages 537-539, New York, NY, USA, 2001. ACM. 